\title{
Influence of Zerumbone Supplementation a Natural Dietary Product from Zingiber zerumbet smith on Early-Developed Atherosclerotic Lesions in Cholesterol-Fed Rabbits
}

\author{
Hemn H.O ${ }^{1,3, *}$, Hazilawati $\mathrm{H}^{1}$, Noordin M.M ${ }^{1}$, Heshu S.R ${ }^{1,3}$ and Zuki A.B.Z ${ }^{2}$ \\ ${ }^{I}$ Department of Pathology and Microbiology, ${ }^{2}$ Department of Preclinical Sciences Faculty of Veterinary Medicine, \\ Universiti Putra Malaysia, 43400 UPM, Serdang, Malaysia \\ ${ }^{3}$ Department of Histopathology and Anatomy, Faculty of Veterinary Medicine, University of Sulaimanyah, Sulaimanyah \\ City, Kurdistan Region, Northern Iraq
}

\begin{abstract}
Atherosclerosis is a condition of the arterial wall to 'injury', which is prominently driven by inflammatory and proliferative responses. The objective of this investigation is to evaluate the impact of Zerumbone (ZER), a natural cyclic sesquiterpene isolated from Zingiber zerumbet Smith against early-developed atherosclerotic lesion in the aorta of New Zealand white rabbits fed a cholesterol-rich diet.

Forty two male rabbits used in the present study were randomly assigned into seven experimental groups as follows: EG-I (CN) considered as control group was fed a standard rabbit pellet diet, EG-II (CP) given a cholesterol-rich diet (1\%), EGIII $(\mathrm{Z}+\mathrm{CP})$ given ZER $(0.4 \%)$ two weeks before day 0 as preventive measure and thereafter with the course of cholesterolrich diet, EG-IV $(\mathrm{Z} 1+\mathrm{CP})$ given ZER $(0.8 \%)$, EG-V $(\mathrm{Z} 2+\mathrm{CP})$ given ZER (1\%), EG-VI (S+CP) given Simvastatin (SIM) $(20 \mathrm{mg} / \mathrm{Kg})$ and EG-VII $(\mathrm{S}+\mathrm{Z}+\mathrm{CP})$ given ZER $(0.8 \%)$ together with SIM $(20 \mathrm{mg} / \mathrm{Kg})$. Experimental groups from EG-IV to VII believed to represent the therapeutic measure of ZER against early-developed atherosclerosis. Tissue samples were collected from the thoracic aorta and aortic arch at 12 weeks post-feeding for gross, histopathological and ultrastructural (scanning electron microscopy) examination.

Morphological analysis (both microscopic and ultrastratural) revealed that endothelial damage and atheromas plague built up were notably diminished in ZER treated groups in a dose dependent manner where it is more prominent in EG-III and EG-VII compared to EGs-VI, V, and VI. However, most of the ZER treated groups showed obvious reduction in plague development in contrast to cholesterol-rich diet group EG-II.

Our data indicate that dietary intake of ZER considerably averts and decreases early plague formation and development via significant lessening in smooth muscle cells proliferation and reduction of inflammatory progression.
\end{abstract}

Keywords: Rabbits, Hypercholesterolemia, Atherosclerosis, Zerumbone, Histopathology, Ultramorphology.

\section{INTRODUCTION}

Cardiovascular disease is one of the major health care problems and the most common cause of death among individuals from developing nations. Atherosclerosis results in cardiovascular death of approximately 16.7 million people around the world each year (WHO) health report in 2003 [1]. It is a vascular disease of greatest importance in human beings, occurs only infrequently in animals and rarely leads to clinical diseases. Lesions of the naturally occurring disease have been detected in aged pigs and birds and in dogs with hypothyroidism, which develops an accompanying hypercholesterolemia [2].

Herbs and spices are generally considered safe and proved to be effective against various human ailments. One of these traditional plants is Zingiber Zerumbet Smith extract, which is known as Zerumbone (ZER) belongs to the

*Address correspondence to this author at the Department of Pathology and Microbiology, Malaysia; Tel: +6012-2471829;

E-mails: himenho75@yahoo.com family of (Zingiberaceae). ZER (1, 2,6,9-humulatrien-8-one, or 2,6,9,9- tetramethyl-[2E,6E,10E]-cycloundeca-2,6,10trien-1) is a cyclic sesquiterpene found as the main component of the essential oil of a wild ginger, Zingiber zerumbet Smith [3].

The compound has been reported to have antiinflammatory and antiproliferative potential. Moreover, the compound has been implicated as one of the most promising chemopreventive agent against colon and skin cancer [4-7].

Hence, the present study was designed to evaluate the antiproliferative effect of ZER against developing atherosclerotic lesion in the aorta of long term feeding of cholesterol in male albino rabbits through morphological analysis (gross, microscopic and ultrastratural changes).

\section{MATERIALS AND METHODS}

\section{Animals and Experimental Design}

Forty two male New Zealand white rabbits 10-12 weeks old used in the present study were randomly assigned into seven experimental groups as follows: EG-I considered as 

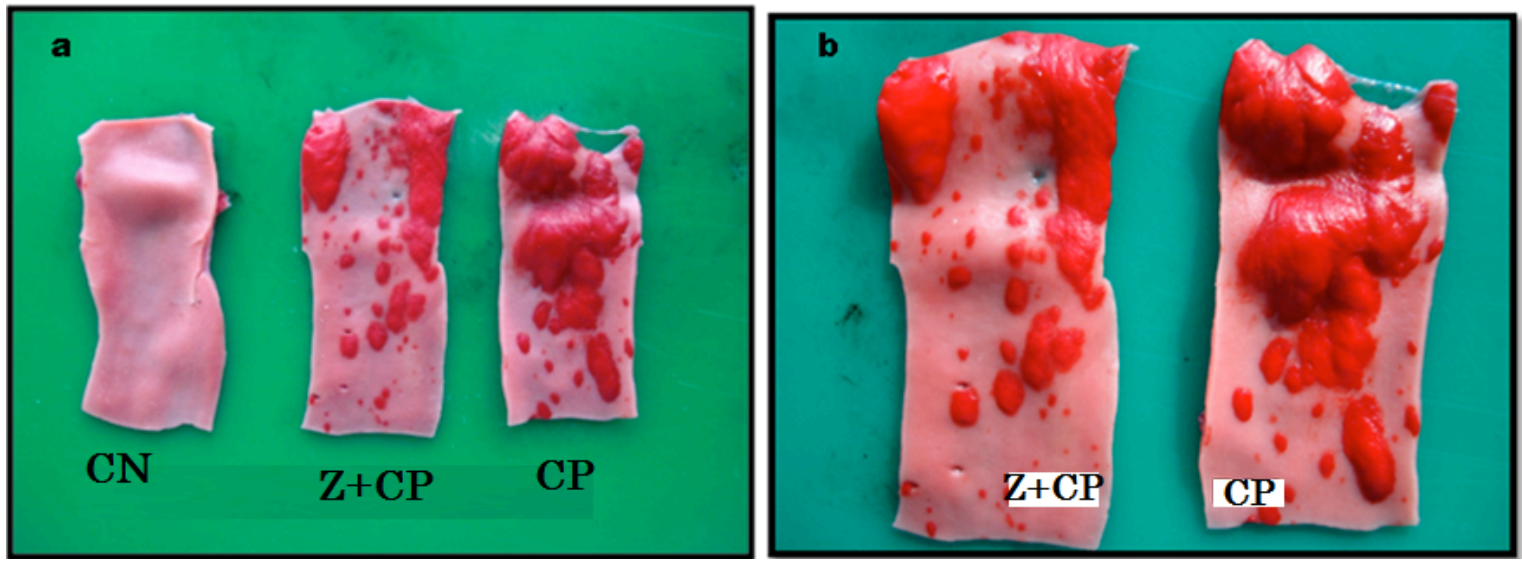

Fig. (1). (a, b) Photograph of the intima of the aorta following staining by Sudan IV. Note marked bright red lipid deposits in CP compared to other groups.
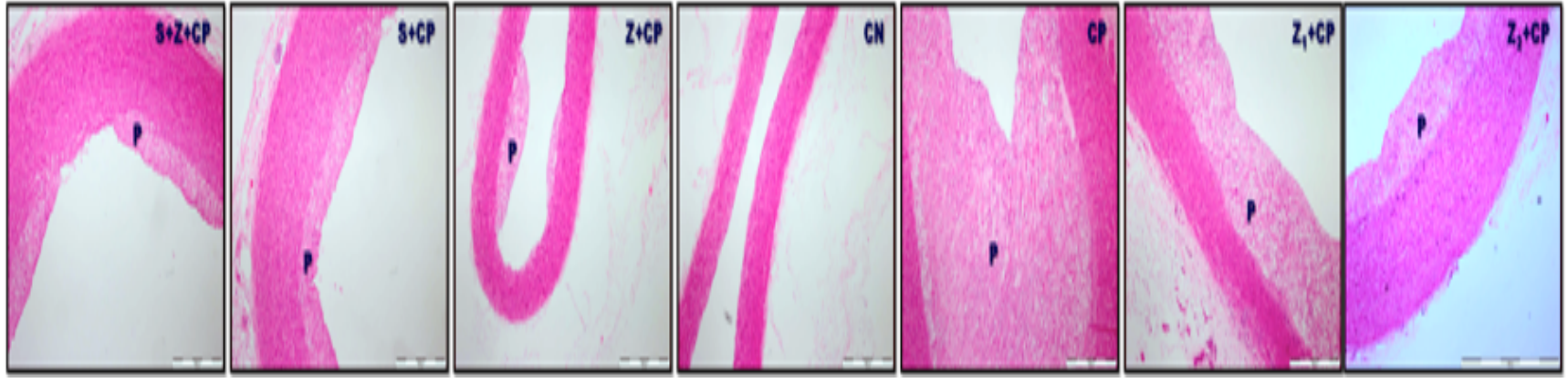

Fig. (2). Photomicrograph of the thoracic aorta of all groups at the end of the experiment as stained by H\&E. Scale bars: $500 \mu \mathrm{m}(\mathrm{CN} \&$ $\mathrm{Z}+\mathrm{CP}), 200 \mu \mathrm{m}\left(\mathrm{CP}, \mathrm{Z}_{1}+\mathrm{CP}, \mathrm{Z}_{2}+\mathrm{CP}, \mathrm{S}+\mathrm{CP}\right.$ and $\left.\mathrm{S}+\mathrm{Z}+\mathrm{CP}\right)$.

control group was fed a standard rabbit pellet diet, EG-II given a cholesterol-rich diet (1\%), EG-III given ZER $(0.4 \%)$ two weeks before day 0 as preventive measure and thereafter with the course of cholesterol-rich diet, EG-IV given ZER $(0.8 \%)$, EG-V given ZER (1\%), EG-VI given Simvastatin (SIM) $(20 \mathrm{mg} / \mathrm{Kg})$ and EG-VII given ZER (0.8\%) together with SIM (20mg/Kg).

Experimental groups from EG-IV to VII acted to represent the therapeutic measure of ZER against earlydeveloped atherosclerosis. The rabbits were euthanized by an overdose of sodium pentobarbital via intra-cardial injection at the end of 12 weeks, and aortas were removed for assessment of atherosclerotic plaques and morphological investigation.

\section{Assessment of Atherosclerotic Plaques}

The tissues were immersed in Herxheimer's solution that contained 5 g Sudan IV, $500 \mathrm{~mL}$ of $70 \%$ ethyl alcohol, and $500 \mathrm{~mL}$ acetone at room temperature for 15 minutes. The aortas were then transferred to $80 \%$ alcohol for 20 minutes, washed in running water for 1 hour, and then mounted and photographed. Aortic atherosclerosis was determined by distribution of sudanophilia in the photographs and expressed as percent of aortic area.

\section{Histopathological Evaluation}

Tissue samples (aortas) were removed and fixed in 10\% NBF (100 ml formalin (40\%), $4 \mathrm{~g}$ sodium phosphate monobasic, $6.5 \mathrm{~g}$ sodium phosphate dibasic and $900 \mathrm{ml}$ D.W) and processed for paraffin embedding. The histopathological sections $(4-6 \mu \mathrm{m})$ were stained with haematoxylin and eosin.

\section{Scanning Electron Microscopy (SEM) Analysis}

Samples were dehydrated through a gradually increasing acetone series $(35 \%, 50 \%, 70 \%, 95 \%$ and $100 \%) 15$ minutes for each. The specimens were placed into specimen basket and kept in amyl acetate for critical point drying (BALTEC CPD 0301 Japan) for about 1 hour and half. Tissues were coated with gold-palladium particles in Sputter coater (BALTEC SCD 005 Japan) and examined using scanning electron microscope (Philips XL30 ESEM (Germany).

\section{RESULTS AND DISCUSSION}

\section{Sudanophilia Evaluation}

Gross vertical dissection to the aortic arch and thoracic aorta, showed, aortic-intimal fatty streaks after Sudan IV staining, in which these lesions appeared as flat or very slight orange to red elevated intimal-medial streaks and spots of sharply demarcated sudanophilia. At the end of experimental period 12 weeks, Sudan IV stained lesions showed significant reduction in the growth of area of sudanophilia in EG-III $(\mathrm{Z}+\mathrm{CP})$ in compare to EG-II (CP). On the other hand, no lesions were detected in $\mathrm{CN}$, (Fig. 1a and $\mathbf{b}$ ).

\section{Histopathological Evaluation}

Sections from the thorasic aorta revealed marked elevation of atherosclerotic plaque (p) which completely occupied the intimal layer and the arterial lumen in CP group 

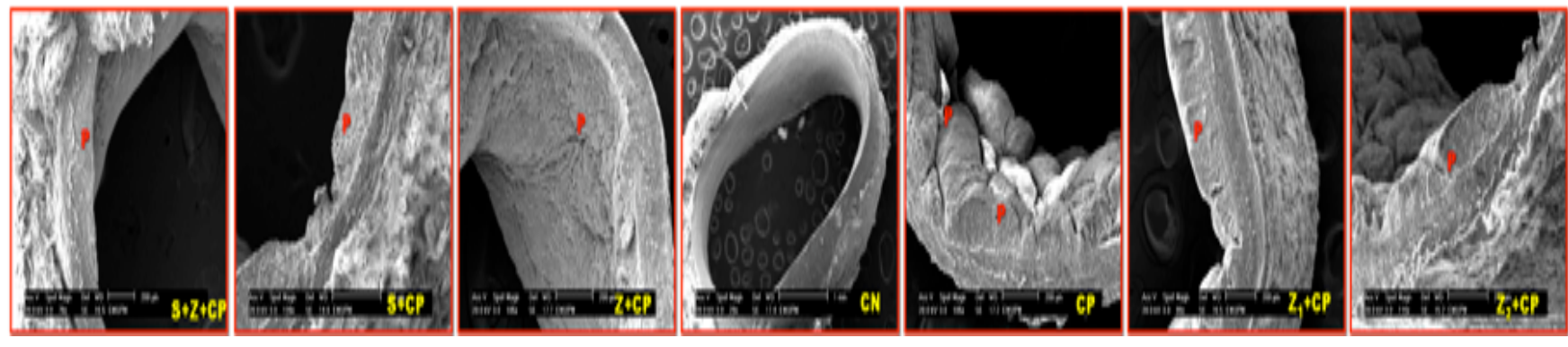

Fig. (3). Electromicrograph of different treatment group of thorasic aort.Scale bars: $500 \mu \mathrm{m}(\mathrm{CN}$ gp), $200 \mu \mathrm{m}$ gps $(\mathrm{CP}, \mathrm{Z}+\mathrm{CP} \mathrm{Z} 1+\mathrm{CP}$, $\mathrm{Z} 2+\mathrm{CP}, \mathrm{S}+\mathrm{CP}$ and $\mathrm{S}+\mathrm{Z}+\mathrm{CP}$ ).

in compare to $\mathrm{CN}$ group. However, there is noticeable averting in plaque formation in $\mathrm{Z}+\mathrm{CP}$ group in comparing with CP gp (Fig. 2)(These experimental groups represent the protective efficacy of ZER in preventing the formation of atheromas plaque). Furthermore, There is an apparent lessening in plaque development in groups $\left(Z_{1}+C P, Z_{2}+C P\right.$, (Fig. 2) and gp ( $\mathrm{S}+\mathrm{Z}+\mathrm{CP})$ (Fig. 2) in compare to $\mathrm{CP}$ group in a dose dependent manner where it is more prominent in groups $\left(Z_{2}+C P\right.$ and $\left.S+Z+C P\right)$ in compare to groups $\left(Z_{1}+C P\right.$ and $\mathrm{S}+\mathrm{CP}$ ) (Fig. 2). Nevertheless, still not significant in compare to $\mathrm{CN}$ gp (These experimental groups represent the therapeutic efficacy of ZER in reducing the development of atheromas plaque).

\section{Scanning Electron Microscopy Evaluation}

Electromicrograph of the thorasic aorta exposed multiple lipid-filled variable sized elevation of atherosclerotic plaque (p) on the intimal surface in $\mathrm{CP}$ group in compare to $\mathrm{CN}$ group. However, there is clear prevention in plaque structure in $\mathrm{Z}+\mathrm{CP}$ group in comparing with $\mathrm{CP}$ gp (Fig. 3) (These experimental groups represent the protective efficacy of ZER in preventing the formation of atheromas plaque). Furthermore, There is an visible reduction in plaque development in groups $(\mathrm{Z} 1+\mathrm{CP}, \mathrm{Z} 2+\mathrm{CP}, \quad$ (Fig. 3) and $\mathrm{S}+\mathrm{Z}+\mathrm{CP}$ ) (Fig. (3) in compare to $\mathrm{CP}$ group in a dose dependent manner where it is more prominent in groups $(\mathrm{Z} 2+\mathrm{CP}$ and $\mathrm{S}+\mathrm{Z}+\mathrm{CP}$ ) (Fig. (3) in compare to groups $(\mathrm{Z} 1+\mathrm{CP}$ and $\mathrm{S}+\mathrm{CP})$. Even so, still not significant in compare to $\mathrm{CN}$ gp (Fig. (3) (These experimental groups represent the therapeutic efficacy of ZER in reducing the development of atheromas plaque).

This study demonstrated that dietary consumption of ZER in hypercholesterolaemic rabbits significantly reduced or in some extent prevents the development of aortic atherosclerotic lesions these effects predominantly due to the anti-proliferative and anti-inflammatory outcome of ZER against macrophage aggregation. Arterial wall macrophages play a major role during early atherogenesis. Oxidative stress induces macrophage responses leads to increased capacity to oxidize LDL, increased Ox-LDL cellular uptake, as well as macrophage lipid peroxidation and foam cell formation in the wall of affected artery. Aggregated LDL is taken up by macrophages at enhanced rate, leading to cellular cholesterol accumulation and foam cell formation [8].

It is evident from the results that feeding ZER along with cholesterol for 90 days resulted in less marked hyperlipidemia (data not showed) and comparatively lowers grades of atherosclerosis in the aorta as compared to the pathogenic control group, i.e. the group maintained on cholesterol diet alone., suggested that ginger stimulate the conversion of cholesterol to bile acids, an important pathway of elimination of cholesterol from the body [9].

Moreover, the reduction in atherosclerosis lesion and plaque built up may be due to the anti-inflammatory antioxidant effect of ZER in suppression mononuclear inflammatory cell migration via suppression of COX-2 expression and inducible nitric oxide [10], furthermore, elimination of free radicals through reduction in inflammatory cytokines such as interleukin-1 and TNF- $\infty$ through the inhibition of NF- $\kappa \mathrm{B}$ activation [11].

\section{CONCLUSION}

The results of present experimental study indicate that consumption of ZER may be proven beneficial in attenuation of atherosclerosis development, since it is associated with reduced macrophage-mediated oxidation of LDL, reduced uptake of oxidized LDL by macrophages, reduced oxidative state of LDL by eliminating free radicals and reduced LDL aggregation. All these effects lead to a reduced cellular cholesterol accumulation and foam cell formation, the hallmark of early atherosclerosis. However, ZER may not be as effective in humans in reducing serum cholesterol levels or in repairing endothelial damage as observed in rabbits. Further studies including more subjects are needed to better understand the effects of ZER on cardiovascular disorders.

\section{CONFLICT OF INTEREST}

The authors confirm that this article content has no conflicts of interest.

\section{ACKNOWLEDGEMENTS}

Declared none.

\section{REFERENCES}

[1] Elena, G.; Klaus, L. Vascular Adhesion Molecules in Atherosclerosis. Arterioscler Thromb. Vasc. Biol., 2007, 27, 22922301.

[2] McGavin, Md., Carlton, W.W., Zachary, J.F. Thomson's Special Veterinary Pathology. Mosby, Inc: Philadelphia U.S.A 2001.

[3] Xian, M.; Ito, K.; Nakazato, T.; Shimizu, T.; Chen, C.K.; Yamato, K.; Murakami, A.; Ohigashi, H.; Ikeda, Y; Kizaki, M. Zerumbone, a bioactive sesquiterpene, induces G2/M cell cycle arrest and apoptosis in leukemia cells via a Fas-and mitochondria-mediated pathway. Cancer Sci. 2007, 98(1), 118-126.

[4] Kitayama, T.; Okamoto, T.; Hill, R.K.; Kawai, Y.; Takahashi, S.; Yonemori, S.; Yamamoto, Y.; Ohe, K.; Uemura, S.; Sawada, S. 
Chemistry of zerumbone. 1. Simplified isolation, conjugate addition reactions, and a unique ring contracting transannular reaction of its dibromide. J. Org. Chem., 1999, 64(8), 2667-2672.

[5] Murakami, A.; Tanaka, T.; Lee, J.; Surh, Y.; Kim H. Zerumbone, a sesquiterpene in subtropical ginger, suppresses skin tumor initiation and promotion stages in ICR mice. Int J. Cancer, 2004, 110, 481490.

[6] Murakami, A.; Ohigashi, H. Cancer-preventive anti-oxidants that attenuate free radical generation by inflammatory cells. Biol. Chem. 2006, 387, 387-392.

[7] Sadhu, S.K.; Khatun, A.; Ohtsuki, T.; Ishibashi, M. First isolation of sesquiterpenes and flavonoids from Zingiber spectabile and identification of zerumbone as the major cell growth inhibitory component. Nat. Prod. Res. 2007, 21(14), 1242-1247.

[8] Aviram, M. Modified forms of low density lipoprotein and atherosclerosis. Atherosclerosis, 1993, 98, 1-9.

[9] Sharma, I.; Gusain, D.; Dixit, V.P. Hypolipidemic and antiatherosclerotic effects of Zingiber officinale in cholesterol fed rabbits. Phytother Res. 1996, 10, 517-8.

[10] Murakami, A. Chemoprevention with phytochemicals targeting inducible nitric oxide synthase. Forum. Nutr. 2009, 61, 193-203.

[11] Murakami, A, Takahashi D, Koshimizu K, Ohigashi H. Synergistic suppression of superoxide and nitric oxide generation from inflammatory cells by combined food factors. Mutat. Res., 2003, $523,151-161$.

Received: May 29, 2013

Revised: September 07, 2013

Accepted: October 03, 2013

(C) Hemn et al.; Licensee Bentham Open.

This is an open access article licensed under the terms of the Creative Commons Attribution Non-Commercial License (http://creativecommons.org/licenses/by$\mathrm{nc} / 3.0 /$ ), which permits unrestricted, non-commercial use, distribution and reproduction in any medium, provided the work is properly cited. 